\title{
Trend of Internet of Things industry in China under the Globalization
}

\author{
Fengqin Sui ${ }^{1, a}$ \\ ${ }^{1}$ University of Science and Technology LiaoNing, China \\ a765914980@qq.com
}

\begin{abstract}
Keywords: Internet of things (loT).
\end{abstract}
Abstract. Under the impact of the new generation of information and communication technology, China is speeding up things to other areas of urban management, new energy, healthcare, manufacturing penetration. Especially in recent years, developed countries and regions have introduced policies of things related to the strategic layout, hoping to seize the opportunities in the new round of development of the information industry. With the maturity of industrial applications and look forward to a new technology platform versatility of things it occurs in China.

\section{Introduction}

Internet of things is an important part of a new generation of information technology, It is also an important stage in the development of "information" age. Its English name is: "Internet of things (IoT) "。As the name suggests, Internet of things that material objects connected to the Internet. This has two meanings: First, The core and foundation of IoT is still the Internet, The Internet is based on the extension and expansion of the network; Second, Between extended and expanded its client to any goods and articles, Information exchange and communication, That is physical objects interest. IoT is an application to expand the Internet, IoT are not so much a network, As it is a business and networking applications.

\section{Internet of things conditions of industrial development}

To make IoT have the greatest economic impact, we must have certain conditions, and to overcome some difficulties. Some are technical problems, some question the organizational structure, business management and other aspects of government regulation. In this regard, the World Institute on the basis of the computer industry on the investigation, summarizes eight key factors affecting the success of the development of IoT:

1.The technical conditions. For a more extensive use of the Internet of Things, we must continue to reduce the cost of the underlying hardware. Therefore, low-cost, low-power sensor is essential. To give users access to things most of the data, and use more data, computing and storage costs must also continue to decline, and the analysis and visualization software technology also needs to be further developed.

2.Security and privacy protection. Things appeared to bring more equipment, which has turned into a potential network vulnerabilities a node. Look for these vulnerabilities and eliminate security risks is one of the key tasks of things.

3.Protection of intellectual property. To release the full potential of the Internet of things, the need to reach consensus on a variety of connected devices generated data ownership. For the sensor data produced by a company, and obtained by the data belonging to third parties deployed in the environment part of the solution, data ownership needs to draw.

4.Enterprise change management system. Companies need their organizations, partners and re-operations planning. With the influx of more data, companies need to establish a decentralized organizational structure, decision-making power devolved to frontline staff. Enterprises need to establish a new organizational structure, support staff and business partners for other departments to carry out more creative cooperation. 
5.And standardization. To realize the full value of networking applications, networking equipment and systems ability to work together is critical; if there is no interoperability, at least $40 \%$ of the potential benefits can not be achieved. Open standards is one way to achieve interoperability. 6.Energy security. When the power supply is still one of the issues of most concern modern data center. Things environmental future success of electrical energy must be taken into account, because it requires the deployment of tens of billions of devices, sensors, and related infrastructure. According to Gartner, by 2020 this number could reach 1.9 trillion. Energy saving technology will be one of things developing with new economic value.

7.Government policy and regulation. Things have to subvert the "rules of the game" capability, but in the new situation will give rise to new rules, new processes, new decision-making and even the new regulatory system. A well-governed networking platform will contain the following five principles: open, participation, accountability, effectiveness and unity. In addition, policy makers often influence in shaping the Internet of Things market rules applied plays a role, such as the establishment of appropriate incentives in health care. Finally, the government can also for things data collection, sharing and use of data to develop practical rules and regulations.

8.Organization and personnel management. Things will combine the physical and digital worlds together to challenge the traditional notion of responsibility organization. Things in the world, IT and physical assets and inventory tightly linked, directly affect the business operations indicators measured, these functions must be more closely united. In addition, the company not only need to have things how the system works (by employee or partner / supplier relationships), and requires the use of the Internet of Things to guide data-driven decision-making ability and mentality, and to allow their organizations to adapt to new processes and business art modes.

\section{Internet of things developed Development Status}

Governments concerned, the development of the networking industry can improve the overall national strength and the socio-economic benefits, bringing huge industrial cluster effect, to play an important role in the future of intelligent society. Networking for this matter in the United States to upgrade the concept of national development strategies, and then followed by the countries in the world have to develop their own national development strategy, marking things into the historical process of development of human society. In the United States, the United States is to provide a blueprint for the development of things by the companies to develop and expand in its core technology fully mature conditions. United States in November 2008, IBM announced the strategic wisdom of the earth, "wisdom of the Earth" to propose "embedded sensors and equipment to the power grid, railways, bridges, tunnels, highways, buildings, water systems, dams, oil and gas pipelines the object, and is generally connected to form a so-called Internet of things, and by supercomputers and cloud computing will integrate things together to achieve social and physical systems integration. "January 2009, IBM and the US think tank Information Technology and Innovation Foundation (ITF) jointly submitted to the Obama administration's "The Digital Road to Recover A Stimulus Plan to Create Jobs, Boost Productivty and Revitalize America", Presented by information and communication technology (ICT) investment can create jobs in the short term, and long-term development while driving the United States, which encouraged the development of networking technology policy is mainly reflected in the promotion of energy, broadband and three medical field of application of networking technology. As a higher stage of information technology, the US government did not hesitate to put forward the strategic goal of the development of things, and on the policy vigorously support the networking industry, to ensure that the United States maintain its leading position in the next phase of international economic competition.

\section{Characteristics of the global development of the Internet of Things industry}

At present, the development and application of networking technology mainly in the United States, Europe, Japan, Korea and a few other developed countries. By analyzing the United States, Japan, Europe, Korea and the networking industry can be seen, the global development of things show the following main features: 
1.From the geographical pattern to see in the next decade, the developed economies will likely create more value things, because of the higher value associated with each of its deployment. However, the developing economies of Things applications there is also great potential value. In emerging markets, compared to Russia, India and Brazil, China is expected to achieve a more significant economic benefits from the industrial Internet of Things.

2.From the temporal pattern, the global networking stage of accelerated development as a whole, the next few years, the global networking market will emerge rapidly. Things the chain enterprise resource investment continued to increase. Base semiconductor giants have introduced to the networking technology needs special chip products for the rapid development of the whole industry to provide a huge boost.

3.From the development of ideas of things to see, there is a big difference between developed and developing countries. Things in the developed markets (such as Europe) presents the laws, regulations, policies, standards, technologies, applications gradually promote the development of ideas. The basis of this important development ideas is a powerful research and development efforts in Europe and America the world's top 500 enterprises, the networking technology innovation to become a source of networking industry sustained rapid development. In developing countries, the networking industry is not mature, its high-end technology, there is a distinct disadvantage, which limits the application process things.

4.The development of things from the technical conditions, the Internet is one of the basis for the development of things, the relationship between the degree of development of the Internet of Things development process. The development of the Internet in each country the situation is different. In the rapidly growing popularity of the Internet of Things industry, the United States, Switzerland, the Nordic countries and the Netherlands ahead. Wherein the degree of information of the United States living in the world, according to forecasts, 2012 - May 2016 the United States the number of users accessing the Internet through mobile devices will increase to 174 million from 265 million, the United States can see things developing infrastructure has been basically mature. Spain, Italy, Russia, India and Brazil and other countries is the lack of effective conditions for the development of the Internet of Things industry, mainly due to poor infrastructure, lack of skills, institutional infrastructure is weak, and therefore can not support the extensive application of new technologies.

\section{Internet of things industry development trend of China}

1. Chinese networking industry is developing applications for the pilot, there is a market from public administration and services to business, industry application market, to the personal home market gradually mature market segments progressive trend.

Currently, the networking industry in China is still in the early stage and introducing the concept of industry chain gradually forming stage, there is no mature technology standards and improve the technical system, the whole industry is in its embryonic stage.

2. The standard system of things is the development of a gradual maturing process, it will be presented from the formation of mature applications refining industry standards to drive the industry standard key technical standards, evolutionary trends standard system formed.

Things concept covers numerous technologies, many industries, in many areas, attempting to develop a universal uniform standards almost impossible. Standard networking industry will be a very broad coverage of the standard system, with the gradual development of the market will develop and mature. In the process of industrial development of things, advanced single technology does not necessarily guarantee a certain standard dynamic and vitality, openness and the size of the standard facing the market is to continue its critical and core issues. With networking applications and gradually expand the market matures, which apply greater market share, derived from the application of the relevant standards will be more likely to become widely accepted de facto standard.

3. With the maturity of industrial applications, the versatility of the new networking technology platform will appear.

Things innovation is the integration of innovative applications, a single enterprise can not be completely independent of the completion of a complete solution, a mature technology, improve 
service, many product types, applications, user-friendly application, will be the equipment provider , technology solution providers, operators, service providers result of collaboration. As the industry matures, support different device interface, different network protocols and services can integrate a variety of common technology platform will be the networking industry proven results.

4. Business model innovation for the Internet of Things will be the result of the technical and human behavior fully integrated.

Things your mobile robot are interconnected with society, new business models will emerge to be the result of things related to technology and human behavior fully integrated. China has the world's leading manufacturing capability and industrial base, has a long history of five thousand years of culture, the Chinese people have a rational logic and artistic flexibility of both personality and behavior traits, expect certain things in the field in China can lead the world to produce new business model.

\section{Acknowledgements}

First and foremost, I would like to show my deepest gratitude to Professor X.J. LU. Conversations with him have been most helpful. I shall extend my thanks to Mr. Yu for all his kindness and help. This work has been supported by The Applied school of University of Science and Technology Liaoning.

\section{References}

[1] China Industry Research Network, China Internet of Things market depth investigation and analysis of development prospects(2014)

[2] China Industrial Information Network, Global networking industry development status and future development trend analysis(2015)

[3] China Communication Network, IOT industry trends(2015) 